\title{
Responsabilidad Social y Educación en Honduras
}

\author{
Lenin Leonardo Banegas Barahona \\ leonardo.banegas80@gmail.com
}

\section{RESUMEN}

Las relaciones entre la educación y la responsabilidad social son cada día evidentes derivando en al menos tres vínculos; La responsabilidad social ejecutada por las empresas por su participación en el desarrollo comunitario específicamente en el sector educación, es decir las inversiones socialmente responsables que la empresa privada puede realizar a favor de la educación. La educación y formación de Directores de Responsabilidad Social Empresarial, lo cual es incluyente del desarrollo de propuestas en donde el tema de responsabilidad social empresarial se tome de forma transversal o se generen espacios educativos propios, así como el desarrollo de carreras específicos asociada al tema. La responsabilidad social de los centros educativos en participar en procesos de potenciación del progreso social, económico, ambiental e institucional, como un ciudadano organizativo más, bajo un esquema de territorios socialmente responsables en donde participen todos por el bien común.

En este trabajo se resumen las experiencias de Honduras, en los tres vínculos estableciendo el grado de avance existente hasta el momento sobre esta temática reflexionando sobre todo sobre las perspectivas que existen de que la vinculación entre educación y responsabilidad social se consolide en el Sistema Educativo Nacional, con ello se aporta en especial al dialogo que desde la academia y especialmente desde la Universidad Pedagógica Nacional Francisco Morazán debe de impulsarse, congruente con sus prioridades y líneas de investigación institucionales, en donde la Responsabilidad Social y Educación ha quedado establecida desde el año 2010.

\section{ABSTRACT}

The relationship between education and social responsibility is evident every day, resulting in at least three links. The social responsibility implemented by the companies because of for their participation in community development specifically in the education sector, that is to say the socially responsible investments that private enterprise can make in favor of education. The education and training of Directors of Corporate Social Responsibility, which is inclusive of the development of proposals in which the issue of corporate social responsibility is taken transversally or generate their own educational spaces, as well as the development of specific careers associated with the topic. The social responsibility of educational centers to participate in processes to promote social, economic, environmental and institutional progress, as a more organizational citizen, under a scheme of socially responsible territories where all participate for the common good. 
Recibido 31 de agosto de 2016 /Aceptado 11 de noviembre de 2016

This paper summarizes the experiences of Honduras in the three links, establishing the degree of progress to date on this issue, reflecting above all on the perspectives that exist that the link between education and social responsibility is consolidated in the National Education System ,With that it is contributed in special to the dialogue that from the academy and especially from the National Pedagogic University Francisco Morazán must be promoted, congruent with its priorities and lines of institutional research, where Social Responsibility and Education has

\section{PALABRAS CLAVE}

Competencias; Educación; Responsabilidad Social; Desarrollo Comunitario; Coordinación Interinstitucional; Inversiones Sociales; Fondos Éticos; Participación.

\section{KEY WORDS}

Skills; education; Social Responsibility; Community Development; Interagency coordination; Social investments; Ethical funds; participation.

\section{INTRODUCCIÓN}

La Responsabilidad Social Empresarial (RSE) es un enfoque de hacer negocios que se ha venido impulsando desde los años 70’s , sin embargo la relación con la educación se ha comenzado a desarrollar recientemente, en el momento en que se reconoce que una de las Responsabilidad Sociales de las Empresas es apoyar al desarrollo comunitario y dentro de este a la educación sobre todo público, de tal forma que se han venido impulsando un conjunto de inversiones socialmente responsables orientadas a fortalecer y mejorar la calidad de la educación.

Para que el enfoque sea pleno se ha planteado desarrollar procesos de formación en responsabilidad social empresarial, enfocados a la formación de capacidades y competencias de los directores de responsabilidad social empresarial, esta es una tarea que pocos centros educativos han comprendido a plenitud de tal forma que se desarrolla la temática de manera optativa y transversal, solamente existiendo una experiencia donde una Universidad hondureña forma en responsabilidad social empresarial con un espacio único, en Honduras aun no existen carreras en este tema en pregrado, postgrado (especialidades, maestrías), por lo que se considera que es una tarea tendiente.

Finalmente, los centros educativos como organizaciones dentro de la sociedad también tienen responsabilidades con sus empleados, con la comunidad y con el ambiente, por lo que examina también el rol que tienen los centros educativos en promover su propia responsabilidad social. 
En este trabajo consideramos a manera de hipótesis:

Que no existe un registro de las inversiones socialmente responsables, por lo que es difícil magnificar la contribución del sector privado al fortalecimiento de la educación pública, ya que el Estado no dispone de registros sobre donaciones y apoyos del sector privado.

La responsabilidad social de los centros educativos se realiza de forma tradicional, no sistematizada ni organizada a través de una unidad como una jefatura, departamento de Responsabilidad Social del Centro Educativo hacia la comunidad.

\section{Antecedentes de la Responsabilidad Social Empresarial}

La Responsabilidad Social Empresarial puede considerarse como un enfoque recientemente aceptado en el pensamiento empresarial moderno, aunque sus orígenes puedan ser rastreados hasta los años 50’s del siglo XX, según (Bonilla, Castillo, \& Morales, 2007, pp.38-42), esto puede situarse en los trabajos y pensamiento propio de (Bowen, 1953, pp. 10-30) como de (Levitt, 1958, pp. 29).

Sin embargo de forma más intensa el enfoque de la Responsabilidad Social Empresarial (RSE), comenzó a profundizarse según (Banegas, 2010, pp. 21-26) en tres referentes importantes:

- El primero de ellos son los principios del reverendo León Sullivan (1922-2001), que fueron elaborados como un "Código de Conducta para las empresas que operaban en el Sur de África”, su legado ha sido inmortalizado y adoptados como principios del pacto global, adoptados por el Sistema de las Naciones Unidas.

- Un segundo referente importante es el desarrollado por el Profesor James Austin, quien escribió el inspirador libro traducido de la versión inglesa (2000) en 2003 titulado "El desafío de la colaboración. Como las organizaciones sin fines de lucro y las empresas comerciales alcanzan el éxito mediante alianzas estratégicas" este libro es ilustrativo ya que plantea que es innecesaria la creación de los brazos sociales de las empresas en forma de fundaciones, en virtud que estas se especializan en sectores importantes de la producción o de los servicios, pero no en prestación de servicios sociales, para los que existen ya instituciones sociales nacionales e internacionales especializadas y con una amplia experiencia institucional (Austin, 2003, pp. 141-157),.

- Finalmente un tercer referente en la temática de RSE, es la teoría de los Stakeholders o partes interesadas desarrolladas por R. Edward Freeman, desarrollada en 1984 como un concepto más amplio que el de los actores sociales (Freeman, 1984, pp. 76), esto ha 
ilustrado formas de actuación para el relacionamiento y el manejo de quejas como de las reclamaciones.

Actualmente se reconoce que las empresas socialmente responsables, desarrollan un conjunto de acciones afirmativas con diferentes partes interesadas, las que se encuentran definidas en cinco áreas, estas son:

1. Empleados o colaboradores en lo relacionado a la inclusión social, como al cumplimiento de las normas de seguridad y salud en ambientes de trabajo, como de los derechos humanos laborales, que están vinculados con los Derechos Económicos, Sociales y Culturales (DESC).

2. Vecinos, contribuyendo al desarrollo comunitario en las dimensiones sociales (educación, salud), bajo el esquema de inversiones sociales, mediante el establecimiento de fondos éticos.

3. Consumidores, vinculado al mercadeo responsable, como la educación del consumidor para fomentar el consumo responsable.

4. Con el Estado, las empresas tienen una responsabilidad fiscal, que permite al Estado cumplir sus propias responsabilidades de inversión social (RSEstatal), pero también apoyando a la gobernabilidad en distintos sectores de interés social.

5. Con el Ambiente, las empresas deben comprometerse a la mitigación de riesgos e impactos, mediante la implementación de Mecanismos de Desarrollo Limpio (MDL) y apoyo a las acciones de conservación y restauración ecológica, como la gestión integral de los recursos (agua, energía).

\section{MÉTODOS Y MATERIALES}

Al tratarse de un informe cualitativo, en el que rescata los siguientes elementos sustantivos de las relaciones entre educación y responsabilidad social:

- Evidenciar los mecanismos de coordinación entre la empresa privada y el sector educación, la forma en que se priorizan las inversiones sociales que las empresas realizan en apoyo al fortalecimiento del sector educación 
- Determinar los espacios de formación y capacitación en el sector educación visto desde la perspectiva de las instituciones educativas y las competencias demandadas por el sector privado en sus perfiles de puesto como Coordinadores de RSE.

- En el marco de conocer las iniciativas innovadoras dentro de las instituciones educativas con respecto a la responsabilidad asumida de contribuir al desarrollo comunitario, la protección ambiental y el bienestar humano, se consultó sobre las formas de trabajo que sean indicativas de la Responsabilidad Social de las Universidades (RSU), expresadas en estructuras organizativas, políticas universitarias, memorias de sostenibilidad.

Para obtener esta información, se ha procedido a la realización de un conjunto de consultas tanto a las organizaciones mercantiles (empresas) como a las organizaciones educativas (centros educativos de distintos niveles-básico, medio y superior-), entes federados (Cámaras de comercio, Federaciones sectoriales) y órganos de dirección en el sector educación a nivel descentralizado y centralizado (Dirección Departamental de Educación de Cortés y Francisco Morazán, dos de los principales departamentos donde se concentra la población hondureña; Direcciones Municipales y Distritales de Educación en el departamento de Cortés, donde se concentra la actividad económica de producción y servicios en Honduras).

Se utilizaron para ello entrevistas estructuradas con preguntas abiertas, las cuales se han tabulado en un conjunto de matrices, que permiten determinar convergencias y divergencias conceptuales como metodológicas, que se presentan en forma de resultados (Mecanismos de coordinación entre empresa privada y sector educación; criterios para priorizar y realizar inversiones sociales sostenibles a través de fondos éticos; espacios de formación y capacitación en responsabilidad social en instituciones educativas según niveles, competencias profesionales demandadas por las empresas y corporaciones para sus perfiles de puesto de Coordinación de la Responsabilidad Social Empresaria; Iniciativas innovadoras de RSU, Políticas de RSU, Memorias de Sostenibilidad de RSU).

\section{Responsabilidad Social y Educación}

De todo la amplitud de los espacios de investigación en Responsabilidad Social, queremos presentar los resultados de la reflexión sobre los vínculos entre el enfoque de RSE con el Sector Educación. Por Responsabilidad Social y Educación, se pueden entender al menos tres procesos: 
- La Responsabilidad Social Empresarial (RSE) o Corporativa (RSC) en apoyo al sector educación, en cumplimiento a su aporte al desarrollo local o de la comunidad.

- La Educación en Responsabilidad Social Empresarial, como parte de la formación y capacitación del nuevo empresariado en este enfoque novedoso. Según (CAPACITARSE, 2009, pp. 32) "El campo educativo cuenta con una atención promedio del $60 \%$ de las acciones de RSE" esto considerando un estudio entre 500 personas de 9 países. Esto debido a que "La educación es, a su tiempo, el medio más utilizado para trabajar en el ámbito comunitario y generar impacto como cambio a través de los programas de RSE”.

- La Responsabilidad Social de las Instituciones Educativas, RSU (en el caso de las Universidades) RESESNU (en el caso de las instituciones de educación superior no universitaria), esto como evolución de los roles de extensión universitaria y vinculación Universidad-Sociedad.

\section{Educación en Responsabilidad Social Empresarial}

Si se parte de la tesis, que todo proceso que conduzcan a cambios en la sociedad, necesitan por una parte, el desarrollo de un conjunto de metodologías de abordaje que inicialmente partan de la experimentación empírica en los sectores sociales y económicos incluyendo dentro de esto a la industria y los servicios, un segundo momento no menos importante para afianzar el cambio social, es el derivado de su inclusión en los procesos formativos.

En el caso particular de la temática de Responsabilidad Social Empresarial, empíricamente se puede advertir que se ha desarrollado una práctica derivada de los consensos internacionales y nacionales sobre las formas de actuación social y ambientalmente responsable de las empresas sobre todo las mercantiles, ya que esto no se advierte todavía con robustez en la empresa pública y en las empresas sociales, se ha impulsado intensamente en la empresa privada.

Sin embargo la segunda condición aún es incipiente y no existen según los resultados que arroja la investigación, que existan consensos, con respecto a las formas de organizar la formación, delineando las lógicas de procesos de enseñanza en espacios educativos antecedentes y consecuentes, y sobre todo en la identificación del perfil de competencias de formación que naturalmente deben de correlacionarse con los perfiles de puesto de coordinación o gerencia de la responsabilidad social empresaria. 


\subsection{Espacios de Formación y Capacitación en Responsabilidad Social}

Educar en responsabilidad social, forman parte de las demandas de la industria y los sistemas de producción sobre las instituciones formadoras de talento humano, sin embargo las experiencias sobre este proceso escasean en el contexto hondureño y centroamericano.

En el contexto internacional existen ofertas formativas de postgrado como de educación continua, ofertadas de forma diversa tanto por las instituciones promotoras (Universidades, Centros de Formación, Fundaciones y Asociaciones Empresariales), como también en estructuras de los espacios educativos (cátedras, seminarios, talleres, cursos de actualización, diplomados), exhibiéndose una diversidad temática, que no siempre sigue lógicas de secuencias de contenidos.

En el caso particular de Honduras, el estado de la educación en responsabilidad social empresarial es el siguiente: La Responsabilidad Social Empresarial en la formación de Administradores de Empresas, Trabajadores Sociales e Ingeniería Industrial, es considerada como remedial, siendo un subtema dentro de los cursos de administración, servicio social, derivándose sobre todo acciones de autoformación y presentación de información sintética derivada de la investigación documental.

Caso excepcional se produce en la Universidad de San Pedro Sula (USAP) que inicialmente ha incluido dentro de la formación de Administradores de Empresas en el grado de Licenciatura ha incluido un espacio educativo propio de RSE a partir del 2012 y ha hecho extensivo su curso de Ética y Responsabilidad Social en todas sus carreras que imparte en el nivel de Licenciatura.

Este evidente descuido por parte de las instituciones formadoras sobre todo en el subsistema educativo superior, en el nivel de pregrado, se repite en el nivel de postgrado a nivel de especialidades, maestrías y doctorados en el área de las ciencias administrativas y educación superior. El Doctorado en Dirección Empresarial de la Universidad Nacional Autónoma de Honduras (UNAH), aprobado en 2012 e implementado a partir del 2014, incluye un Seminario de Responsabilidad Social Empresarial.

Recién en el 2015, la Universidad Tecnológica Centroamericana (UNITEC), ha evidenciado su interés, dentro de los procesos de formación continua y educación permanente de formular un plan de formación de Especialistas en Gestión de la Responsabilidad Social Empresarial, para promover la profesionalización y actualización de todos aquellos profesionales vinculados con procesos de Responsabilidad Social, sin embargo, esto aún es un proyecto en formulación, aún no ejecutado. 
La educación corporativa en Responsabilidad Social, es promovida en Honduras casi de forma exclusiva y monopólica por la Fundación Hondureña de Responsabilidad Social Empresarial (FUNDAHRSE), quien desarrolla cursos de actualización, seminarios, talleres y una Conferencia Nacional de carácter anual, los primeros son exclusivos para las organizaciones socias de la Fundación y en el caso de la Conferencia de RSE, es ampliado, sustentado en conferencias magistrales y exposiciones en stand por parte de las empresas socialmente responsable que son socias de FUNDARHRSE.

Por lo anterior se puede concluir que los espacios de formación en esta nueva área de conocimiento, son incipientes en el caso particular de Honduras. Para orientar de forma correcta el diseño curricular, el primer paso es definir las competencias a ser desarrolladas en los procesos formativos y de capacitación, una aproximación conceptual a las competencias la provee (Argudín, 2005, pp. 142) citando a (UNESCO,1999, pp. 130), que definen, las competencias como “ El conjunto de comportamientos socio afectivos y habilidades cognoscitivas, psicológicas, sensoriales y motoras que permiten llevar a cabo adecuadamente un desempeño, una función, una actividad o una tarea".

Las competencias pueden ser genéricas y específicas, en tanto, se aporta a la construcción de la ciudadanía, con las competencias genéricas y se aporta al desempeño del perfil profesional como especialista con las competencias específicas.

\section{b. Competencias de Gestión en Responsabilidad Social Empresarial}

En base a la consulta realizada con empresas socialmente responsables sobre las competencias genéricas y básicas de un gerente de la Responsabilidad Social Empresarial, se pudo determinar el siguiente perfil de competencias, en los dominios tanto cognoscitivo, procedimental como actitudinales.

A continuación se presentan un conjunto de competencias o capacidades a desarrollarse en el proceso formativo, agrupadas en competencias genéricas, como específicas.

\section{i. Competencias Genéricas}

a. Propone acciones de mejora, basado y fundamentado en procesos diagnósticos de la realidad empresarial.

b. Lidera procesos de negociación con partes interesadas y con directivos empresariales para fomentar acuerdos de colaboración y de trabajo en equipo. 
c. Analiza la realidad y los contextos desde perspectivas variadas para disponer de un mapa mental de afectaciones, riesgos, potencialidades en las que la empresa debe tomar consideraciones cuando gestiona su responsabilidad social empresarial.

\section{ii. Competencias Específicas}

a. Identifica y concretiza líneas del pensamiento estratégico empresarial en temas de responsabilidad social, en áreas de relacionamiento con las partes interesadas, voluntariado corporativo y acciones afirmativas.

b. Diseña Sistemas de Gestión de la Responsabilidad Social Empresarial con orientación a la inclusividad social, el desarrollo comunitario, la conservación y restauración del ambiente.

c. Desarrolla procesos de gestión integrada que permitan la reportabilidad social como la comunicación a través de la concretización de acciones del plan de marketing social.

d. Promueve el pensamiento de la responsabilidad social a otros sectores de la sociedad como las universidades, las organizaciones sociales o del tercer sector (ONG's, Cooperativas, Empresas Asociativas, Fundaciones) como también del Estado para integrar acciones de colaboración que promuevan el bienestar humano y la sostenibilidad en los territorios.

c. Estructura curricular del área de conocimiento de la Gestión de la Responsabilidad Social Empresarial

Basado en la estructura de competencias básicas y genéricas, se puede considerar que la estructura curricular básica de un programa de RSE que considere elementos contemporáneos en esta temática debe de contener al menos las siguientes áreas temáticas o familias curriculares.

Área temática de Pensamiento Estratégico y Planificación de la Responsabilidad Social, en el que se incluyan los fundamentos de la gestión de la RSE, incluyéndose lo relativo a la gestión del voluntariado corporativo y lo contentivo al diseño de políticas y estrategias empresariales de responsabilidad social.

Área temática de Conducción de Programas de Responsabilidad Social Empresarial, incluyendo la gestión ambiental y social de la empresa, caso particular, lo relativo a, participación 
empresarial en el desarrollo comunitario, lo cual es incluyente del manejo de los Stakeholders a través del relacionamiento estratégico.

Área temática de Comunicación de la Responsabilidad Social Empresarial, incluyendo dentro de ello el estudio de los métodos de contabilidad social y elaboración de memorias de sostenibilidad, el mercadeo social o marketing con causa y lo entendido de temas contemporáneos de Responsabilidad Social como por ejemplo los Territorios Socialmente Responsables (TSR), Responsabilidad Social Estatal (RSEstatal), la Responsabilidad Social Universitaria (RSU), la Responsabilidad Social Organizacional (RSO), que particularizan la Responsabilidad Social a otros sectores distintos que la empresa privada o mercantil.

d. Opciones de Gestión Curricular en procesos de Educación en Responsabilidad Social Empresarial

Agregar nuevos conocimientos, actitudes y habilidades en la formación de los nuevos profesionales, a los tradicionales y clásicos cursos, siempre representa un reto, por la complejidad que esto supone. Según (Banegas, 2014, pág. 42-45), para ello existen al menos dos caminos para la gestión de la inserción curricular de las nuevas temáticas.

1. Diseño curricular particular, es decir partiendo de una diferenciación hacia nuevas especializaciones como una profesión nueva, es decir considerando que la Gestión Responsabilidad Social, es un nuevo campo profesional, que debe desprenderse por escisión o por fusión de varias áreas de conocimiento, basándose en la interdisciplina, transdisciplina y la multidisciplina.

2. Transversalización curricular, que supone que la Responsabilidad Social Empresarial es un área de conocimiento afín pero no diferente, por lo que se deben de insertar temáticas y áreas temáticas en los espacios educativos como la administración, la mercadotecnia, la contabilidad, la gestión de la calidad, la gestión del talento humano, desarrollo comunitario, gestión ambiental, entre otras.

La opción que se prefiera, naturalmente derivará en un modelo de actuación particular que impactara en las prácticas profesionales de los egresados, si se considera a la Responsabilidad Social Empresarial como una nueva especialidad de las ciencias sociales y administrativas o si por el contrario se considera como un enfoque integrado en cada una de las disciplinas sociales y económicas, como administrativas.

\section{Responsabilidad Social en Educación}


La Responsabilidad Social en Educación (RSEducación), es uno de los campos preferidos por los empresarios, en principio debido a que este sector es sensible a la opinión pública, aumentando la visibilidad de la empresa socialmente responsable, como segundo criterio por el cual según (CAPACITARSE, 2009,pp.13) considera que esta es una opción preferida por el $60 \%$ de las acciones de RSE, es que existe una concepción de los apoyos que este sector necesita (Infraestructura escolar, capacitación docente, utilería estudiantil-vestimenta, cuadernos, libros, computadoras-, becas), lo cual sin desconocer la importancia y relevancia que esto tiene, no exhibe la complejidad emergente, que aflora de tareas como la conservación de un área protegida, el control de la contaminación ambiental, la mitigación de impactos.

Dentro de la arquitectura legislativa Hondureña, se identifica que en instrumentos de política como el Reglamento General de la Ley Fundamental de Educación (Presidente en Consejo de Ministros, 2014), se deja plasmado de forma normativa el rol de las empresas privadas y su participación en el desarrollo del sector educativo, esto queda plasmado en el siguiente articulado.

Artículo 14. Las empresas, como parte de la sociedad, contribuyen al desarrollo de la educación nacional, mediante:

a. La identificación de las demandas del mercado laboral y la relación de la educación con el desarrollo económico productivo del país;

b. La promoción de alianzas estratégicas con centros educativos, para el fomento de la investigación, el desarrollo tecnológico profesional de los trabajadores y educandos del sistema educativo nacional, que permitan acceder a empleos de mejor calidad;

c. La participación en el desarrollo de servicios y programas educativos y culturales, prioritariamente en el ámbito territorial de su asentamiento, en armonía con su entorno social y natural; $y$,

d. El otorgamiento de facilidades a su personal para realizar o completar su educación y mejorar su entrenamiento laboral dentro del local de trabajo o en centros educativos.

Como se puede advertir de la lectura detallada, el espíritu del legislador sobre el tema de la participación de la empresa privada en el desarrollo del sector educativo, se orienta a coordinar los sistemas de información de gestión del recursos humano, demandas (empresas) y ofertas (sistema 
educativo), el desarrollo de nuevas formas de relación entre las empresas y las universidades como gestoras del conocimiento, la participación en el desarrollo educativo de instituciones en su contexto y ámbito y finalmente una mayor inclusión social de los excluidos de oportunidades de formación, incluidos los empleados de las empresas.

El análisis de la información resultante de las consultas con las unidades descentralizadas en el sector educación (Dirección Departamental, Municipal y Distrital de Educación) y empresas privadas, evidencia al menos que existen prácticas de ejecución coordinadas que son ejemplo de buenas como de malas prácticas.

Una de las buenas prácticas que se identifica es la inserción de varios actores donantes (Cooperación Internacional, ONG's, Empresa Privada) en instancias de coordinación interinstitucional para definir criterios mínimos para favorecer inversiones sociales efectivas en el sector educación, estos espacios aunque carecen de legalidad tienen una legitimidad ampliamente ganada, se autodenominan como Mesa temáticas de educación, Mesa de cooperantes en educación, Grupo de coordinación interinstitucional. La formulación de este tipo de estructuras organizativas, ha evitado yerros, como la duplicidad de funciones, y el potenciamiento o robustecimiento de programas pensados desde la autoridad y la institucionalidad nacional.

Una mala práctica identificada tanto en las consultas a empresas como a las autoridades locales de educación, es la que se provoca cuando la empresa gasta los recursos que ha programado para su RSEducación, y realiza las coordinaciones directas con centros educativos, identificados con escasos criterios de racionalización del gasto, en la mayoría de los casos, ignorando la autoridad local competente en el sector educación (Dirección Departamental, Municipal y Distrital de Educación).

Este yerro trae aparejado que los fondos económicos de la RSEducación se invierten en centros educativos del área urbana, ya que son gastos más visibles y publicitados, favoreciendo la promoción de la inequidad territorial, ya que mientras algunos niños y jóvenes se educan con computadoras, en salones climatizados, y con materiales de estudio, becas y otros beneficios, otros, muchos más, se educan con aulas con techos y mobiliario dañados, en escuelas unidocentes y sin más acceso a la educación que el pizarrón y los lápices marcadores.

Además de estas buenas y malas prácticas, producto de la consulta con empresas privadas y autoridades educativas, se puede advertir que los fondos económicos, ya sea que se consideren 
desde la perspectiva de gasto (identificación con escasos criterios de inversión y más de visibilidad) o de inversión (a través de la coordinación interinstitucional) ¡No se manejan con criterios de sostenibilidad! Por lo que se advierte que existe un desconocimiento generalizado tanto en la empresa privada como en las autoridades locales de los beneficios que podría lograrse con el establecimiento de fondos fiduciarios éticos, de los cuales el contexto internacional ha provisto de importantes lecciones tal como lo identifica (Zicari, 2008, pág. 32), en este contexto se hace necesario que estos procesos puedan promoverse para provecho de la niñez y la juventud que se forma para la profesión y la ciudadanía en el sistema educativo nacional.

\section{Responsabilidad Social de las Instituciones Educativas}

Esta arista de las relaciones entre Responsabilidad Social \& Educación, es la menos desarrollada de todas, y parte de la siguiente premisa, ¡Todos y todas! personas e instituciones dentro de un territorio o comunidad humana, tienen responsabilidades sociales compartidas en promover las mejores condiciones para el bienestar social y ambiental. De esta forma la empresa privada tiene una responsabilidad (RSE o RSC), el Estado también tiene una responsabilidad social (RSEstatal) y las organizaciones sociales también tienen una Responsabilidad Social Organizacional (RSO).

La coordinación y esfuerzo conjunto derivado de las corresponsabilidades de estas instituciones en relaciones Estado-sociedad-mercado en pro del bien común son garantes del mejoramiento de las condiciones de la sociedad y el ambiente natural.

En esta compleja red o tejido social, las instituciones educativas también tienen su rol y responsabilidades sociales a ser asumidas, de hecho en la Ley Fundamental de Educación (Congreso Nacional, 2011), reconoce en su artículo No. 13 al hablar de los principios y valores, que el Sistema Educativo Nacional se adhiere a principios como la equidad e inclusión, la participación, la multiculturalidad e interculturalidad, la responsabilidad ambiental, la transparencia, educación y trabajo. Lo que aquí se advierte es que la responsabilidad social de las instituciones educativas no solo es ya limitada a las acciones de formación de niños y jóvenes, sino la contribución mediante la participación informada y la opinión calificada en la construcción de una mejor sociedad.

El espacio tradicional en el que los centros educativos, sobre todo del nivel medio y superior han volcado estos esfuerzos, es mediante el Trabajo Educativo Social (TES), mediante el cual los jóvenes realizan acciones de promoción del bienestar social enfocándose en labores de conservación

y restauración ambiental, como en acciones afirmativas que potencien una mayor inclusión, esta 
última faceta se ha manejado a través de los programas de alfabetización de adultos, que en el caso de Honduras se deriva operativamente del Plan Nacional de Alfabetización Obligatoria, Atención de Rezago Escolar 2014-2017.

Evolutivamente la RSO de los Centros Educativos, se complejiza y se espera que sea más visible y efectiva a medida que se avanza en los niveles educativos, de tal forma que en el nivel superior, se ha identificado la Responsabilidad Social Universitaria (RSU). En tal sentido y retomando los planteamientos de (Vallaeys, 2008, pp. 10) citado por (Aldeanueva, 2011, pág. 116) las universidades al igual que cualquier otro tipo de organización, generan impactos en su entorno al realizar sus actividades. Tales impactos pueden resultar positivos o negativos, las universidades socialmente responsables serán aquellas que amplíen los impactos positivos de sus acciones y mitiguen los impactos negativos derivada de sus actuaciones.

De tal forma que este enfoque se considera evolutivo, sobre aquellos que plantean que junto a las funciones de docencia, se encuentran las de investigación, pero también las de extensión universitaria, que se han convertirse en procesos de vinculación universidad-sociedad y contemporáneamente en RSU.

El estado actual en Honduras con respecto al tema de RSU, en los 21 centros educativos superiores existentes, es que en 10 de ellos no se evidencian estructuras organizativas relacionadas con la extensión universitaria, la vinculación universidad-sociedad, del resto (11), 7 de ellas tienen programas y estructuras organizacionales de extensión universitaria, 3 de ellas tienen acciones denominadas y basadas en el enfoque de Vinculación Universidad-Sociedad, una de ellas destaca por disponer desde el 2007 un programa de Responsabilidad Social Universitaria (RSU), se trata de la Universidad Tecnológica de Honduras (UTH).

La UTH ha desarrollado una experiencia importante basado en el voluntariado corporativo, la prestación de servicios a la comunidad (becas, consultoría jurídica gratuita para la comunidad, capacitaciones a docentes, estudiantes y autoridades del nivel de educación medio), así como un mercadeo responsable (UTH, 2007). Esta experiencia se ha sistematizado presentándolo como un reporte social, sin que estos, cumplan en sí mismos los criterios y normas del Global Reporting Iniciative (GRI), representando un caminar en la temática en el subsistema de educación superior.

\section{CONCLUSIONES}


Después de haber desarrollado el proceso investigativo, expuesto en síntesis en este artículo, se puede concluir lo siguiente:

1. La educación en responsabilidad social empresarial, navega por una nebulosa, ya que esta se encuentra presente en la formación de profesionales como un subtema tratado como un apéndice y no de forma integral, reconociendo ello como un enfoque contemporáneo y moderno del pensamiento empresarial, que se ha traducido en una diversidad de prácticas realizadas por las empresas.

2. La nebulosa en los mecanismos de educación en RSE, impide que se profesionalice esta ocupación, lo que se traduce en una diversidad de criterios en la implementación de las acciones que las empresas realizan específicamente en su responsabilidad social con el desarrollo comunitario en el sector educación.

3. La Responsabilidad Social en Educación en Honduras, se ha desarrollado con esquemas correctos de racionalización de la utilización de fondos económicos, al formularse e implementarse mecanismos de coordinación interinstitucional como la Mesa de educación, sin embargo aún son muchas las empresas que desarrollan su responsabilidad social con la educación, sin estar incluidos y participar en estos mecanismos de coordinación interinstitucional.

4. La Responsabilidad Social que tienen los centros educativos en su participación en la gestión del desarrollo comunitario, si bien forma parte de la práctica continuada por los centros de educación media y superior a través del Trabajo Educativo Social, que realizan y cumplimentan como requisito aquellos que aspiran a graduarse de estos niveles, sin embargo no se advierte la intencionalidad de crear un programa de desarrollo social, repitiéndose acciones año con año, no contándose con una evaluación de impactos de tales inversiones (en tiempo, recursos económicos y voluntades) invertidas por los estudiantes y sus profesores tutores.

5. En el nivel universitario, de las 20 universidades aprobadas en Honduras, solamente una implementa acciones, con el nombre de Responsabilidad Social Universitaria, la UTH, el análisis de sus prácticas evidencia que su reporte social, no se organiza en base a los criterios del GRI, aunque se tiene el mérito de innovar en estos proceso en el país, donde aún la participación de los centros educativos del nivel superior, no se vive como una función, donde 
quienes lo implementan viven esquemas evolutivos anteriores como la extensión universitaria y la vinculación universidad-sociedad, que carecen de la integralidad que provee la gestión de la RSU.

\section{REFERENCIAS BIBLIOGRÁFICAS}

Aldeanueva, I. (2011). Tesis doctoral: Responsabilidad Social en la Universidad. Estudio de casos y propuesta de despliegue. Malaga, España: Universidad de Malaga. Facultad de Ciencias Económicas y Empresariales.

Argudín, Y. (2005). Educación basada en competencias. Nociones y antecedentes. México, D.F.: Editorial Trillas.

Austin, J. (2003). El desafio de la colaboración. Cómo las organizaciones sin fines de lucro y las empresas comerciales alcanzan el éxito mediante alianzas estratégicas. Buenos Aires, Argentina: Granica. Management.

Banegas, L. (2014). Sistematización de experiencias de inserción temática de la Gestión Ambientalmente Racional de Productos Químicos en los niveles de educación formal y no formal en Honduras. Tegucigalpa, Honduras: Proyecto COPs 2 PNUD GEF SERNA CESCCO.

Bonilla, C., Castillo, C. d., \& Morales, M. (2007). Gestión responsable del negocio. Una perspectiva operacional de la Responsabilidad Social Empresarial. Obtenido de Universidad de Chile: http://www.nexorsu.cl/gestion.pdf

Bowen, H. (1953). Social Responsabilities of the Businessman. New York, EUA: Harper.

CAPACITARSE. (2009). La empresa responsable y el impulso educativo. Webinars de RSE 2009. Buenos Aires, Argentina.

Congreso Nacional. (2011). Ley Fundamental de Educación. Decreto No. 262-2011. Tegucigalpa, Honduras: La Gaceta. 22 de febrero del 2012. No. 32,754.

Freeman, E. (1984). Strategic Management: A Stakeholder Approach. New York.

Levitt, T. (1958). The dangers of social responsability. Harvard Business Review, 36(05), 41-50.

Presidente en Consejo de Ministros. (2014). Reglamento General de la Ley Fundamental de Educación. Tegucigalpa, Honduras: La Gaceta No. 33,533 Septiembre del 2014.

UTH. (2007). Responsabilidad Social Universitaria de la UTH. Obtenido de http://www.uth.hn/rse/

Zicari, A. (2008). Fondos éticos. Factores sociales y ambientales en carteras de inversión. Buenos Aires, Argentina: EDICON. 